\title{
Transverse Oscillations of an Underwater Beam-Cable System
}

\author{
Max Blanco and Philip A. Wilson* \\ * School of Engineering Sciences, University of Southampton, \\ Southampton, UK, SO17 1BJ.
}

\begin{abstract}
An Autonomous Underwater Vehicle refuel station is proposed. The power source is located on board a surface vessel, while the AUV is serviced at depth. The structure which connects the two craft is modelled as a cable-beam. Transverse oscillations of this cable-beam system are investigated through a fourth-order differential equation.
\end{abstract}

Keywords: beams, cables, trigonometry, Bernoulli-Euler equation, transverse oscillation, system identification, analytic solution to fourth-order differential equation

\section{INTRODUCTION}

The eventual aim of this work is to manufacture an AUV refuel or recharge station, as depicted in Fig. 1, in which a surface craft powers a submerged AUV. Several advantages obtain with this concept, among which are productivity and operator safety. The transverse vibration which is analysed here is but one strand of an effort to solve a ship-cable-towfish interaction in an arbitrary seaway.

That the system may be liable to irregular Vortex-Induced Vibrations (VIV) was discovered by Blanco, M. [2010]. A calculation was made of frequency based on the taut string theory of Milburn, D. A. [1983]. That calculation, which revealed the fundamental frequency to be on the order of milliHertz, was problematic because the physics of the taut string do not account for the composite nature of the cable, which is in general a helical arrangement of conductors, insulators and tension members. This paper is an effort to replace the taut string theory with a novel beam-cable theory. It is hoped to ameliorate the accuracy with which the present model response represents reality.

One problem with the physics of the Milburn model may have been in their simple representation of the inertia of the system. The choice is therefore made here to investigate the system as a Bernoulli-Euler beam (Timoshenko, S. P. [1953]). The results of Milburn and of Blanco are repeated here for comparison, and as a result, the units employed here are Imperial; conversion Table 1 is included for ease of reference. Han, S. M., H. Benaroya and T. Wei [1999] modelled the dynamics of beams in tranverse mode with a variety of cantilever and free end conditions. They did not include a dynamic mass condition, but did conclude that the Bernoulli-Euler theory was valid for the conditions of the present problem. Laura, P. A. A., J. L. Pombo and E. A. Susemihl [1974] studied the vibrations of a system similar to the present system, but they were concerned with a clamped end condition. The present system is modelled as a pinned dry end, which presumably

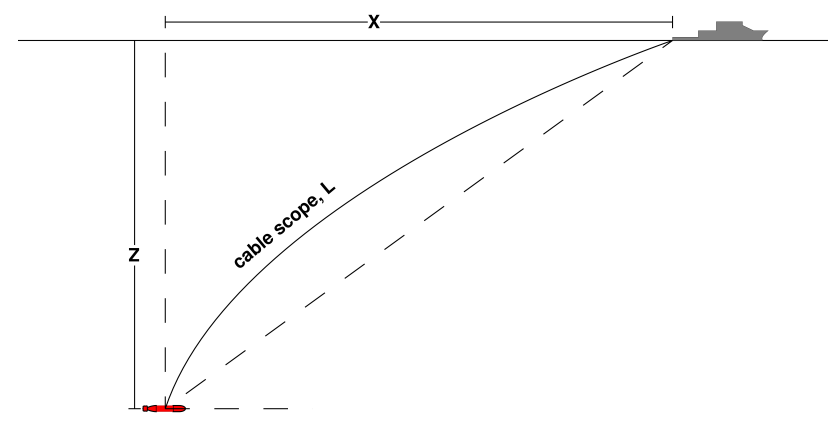

Fig. 1. Depiction and notation for a shipborne AUV refuel station.

has a cleat or a winch termination, and a dynamic masswhich represents the AUV-at the wet end. This problem is amenable to analytic solution, which is the subject of the next section. Section 3 is a report of the numerical calculation. The paper ends with a short summary and outlook.

\section{THEORY}

This section is divided into two subsections. The problem is stated in the first part, and solved in the second.

\subsection{Statement of Problem}

Beams, or bars, in vibration are often modelled by this fourth order differential equation: ${ }^{1}$

$$
E I \frac{d^{4} X(x)}{d x^{4}}-\omega^{2} \frac{A \gamma}{g} X(x)=0
$$

\footnotetext{
1 See Timoshenko, S. and D. H. Young [1955], §51.
} 
where the wavenumber is ${ }^{2}$

$$
k^{4}=\frac{\omega^{2} A \gamma}{E I g}
$$

Prescott, J. [1961] (Ch. 9), Volterra, E. and E. C. Zachmanoglou [1965] (§4.5), Bishop, R. E. D., and D. C. Johnson [1960], Seto, W. W. [1964] and Timoshenko, S., D. H. Young and W. Weaver, Jr. [1974] can be consulted on this subject.

The four boundary conditions imposed on the solution are determined by the physics of the problem, and in turn, determine the solution. This paper models winch or cleat terminations on the ship with pinned end boundary conditions, as follows:

$$
\begin{aligned}
\left.X\right|_{x=0} & =0 \\
\left.\frac{d^{2} X}{d x^{2}}\right|_{x=0} & =0
\end{aligned}
$$

A free mass at the underwater plunger end implies

$$
\begin{gathered}
\left.\frac{d^{2} X}{d x^{2}}\right|_{x=L}=\quad 0 \\
-\left.E I \frac{d^{3} X}{d x^{3}}\right|_{x=L}=\omega^{2} W_{p} X
\end{gathered}
$$

In order of appearance, these are statements that:

- the pinned end is fixed;

- the pinned end is allowed non-zero slope but no curvature;

- the bending moment at the plunger end is identically zero;

- the dynamic shear force at the plunger end is identically the inertial force of the free plunger mass, $W_{p}$.

Equation 4 causes this imagined device to differ substantially from that of Laura, P. A. A., J. L. Pombo and E. A. Susemihl [1974], who investigated a cantilevered beam.

\subsection{Solution of Problem}

Trial solutions are found by substitutions of

$$
\begin{aligned}
X(x) & =C_{1}[\cos k x+\cosh k x]+C_{2}[\cos k x-\cosh k x] \\
& +C_{3}[\sin k x+\sinh k x]+C_{4}[\sin k x-\sinh k x]
\end{aligned}
$$

or (equivalently, see Prescott, J. [1961], §149)

$$
X(x)=A \cos k x+B \sin k x+H \cosh k x+J \sinh k x
$$

into Eq. 1 with Eqs. 3 to 6 as boundary conditions. The equation with the $C_{i}$ subscripts is discounted here because the solution for Eq. 8 is much easier to manipulate in the present instance.

Equation 3 dictates that $A+H=0$ because the cosine and hyperbolic cosine functions are non-zero at $x=0$, while Eq. 4 dictates that $-A+H=0$ for similar reasons, as depicted in Fig. 2. The only way for these equations both to be true is for $A=H=0$.

2 The various quantities are defined in the section on nomenclature, and will not be repeated here.

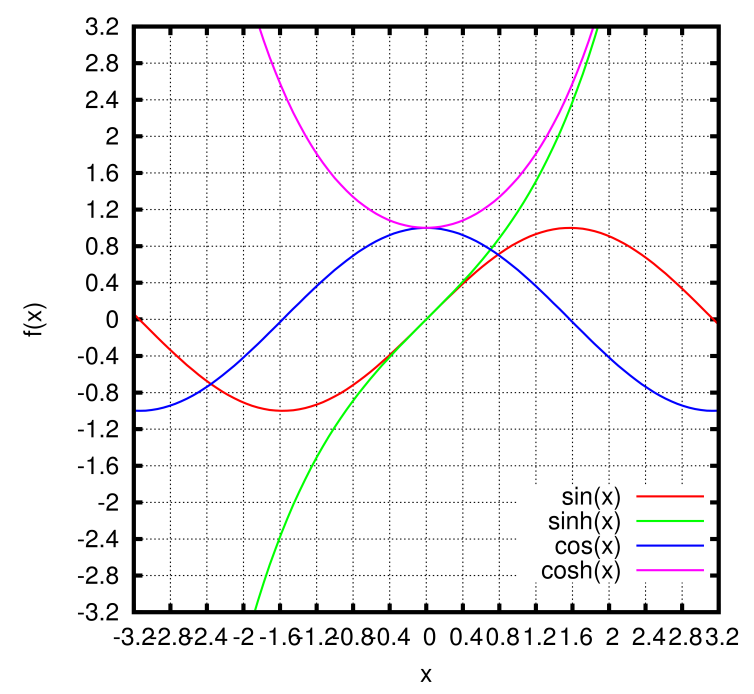

Fig. 2. The four trigonometric functions that contribute to the solution of the fourth-order differential equation for a beam.

Now Eq. 5 gives, at $x=L$

$$
-B k^{2} \sin k L+J k^{2} \sinh k L=0
$$

The second boundary condition at the wet end implies that

$$
\begin{gathered}
+E I B \cos k L-E I J \cosh k L= \\
+\omega^{2} m_{p} B \sin k L+\omega^{2} m_{p} J \sinh k L
\end{gathered}
$$

or

$$
\begin{aligned}
& 0=B\left(E I k^{3} \cos k L-\omega^{2} m_{p} \sin k L\right) \\
& +J\left(-E I k^{3} \cosh k L-\omega^{2} m_{p} \sinh k L\right)
\end{aligned}
$$

Non-trivial solutions of the homogeneous system of Eqs. 9 and 11 must exist in the nolocations ${ }^{3}$ of the determinantal equation. These nolocations, the first few of which are listed in Table 5, are the vibration frequencies for the system.

$$
\begin{aligned}
& 0=-\sin k L\left(-E I k^{3} \cosh k L-\omega^{2} m_{p} \sinh k L\right) \\
& -\sinh k L\left(E I k^{3} \cos k L-\omega^{2} m_{p} \sin k L\right) \\
& 0=\quad 2 \omega^{2} m_{p} \sin k L \sinh k L \\
& +E I k^{3}(\sin k L \cosh k L-\sinh k L \cos k L) \\
& 0=2 \frac{\omega^{2} m_{p}}{k^{3} E I}+\left(\frac{\cosh k L}{\sinh k L}-\frac{\cos k L}{\sin k L}\right) \\
& 0=2 k L \alpha+(\operatorname{coth} k L-\cot k L)
\end{aligned}
$$

where $\alpha$ is the ratio of the mass of the plunger to the mass of the beam-cable. Figure 3 depicts a segment of Eq. 15 when $\alpha=1$.

\footnotetext{
3 literally, locii of the zeros
} 


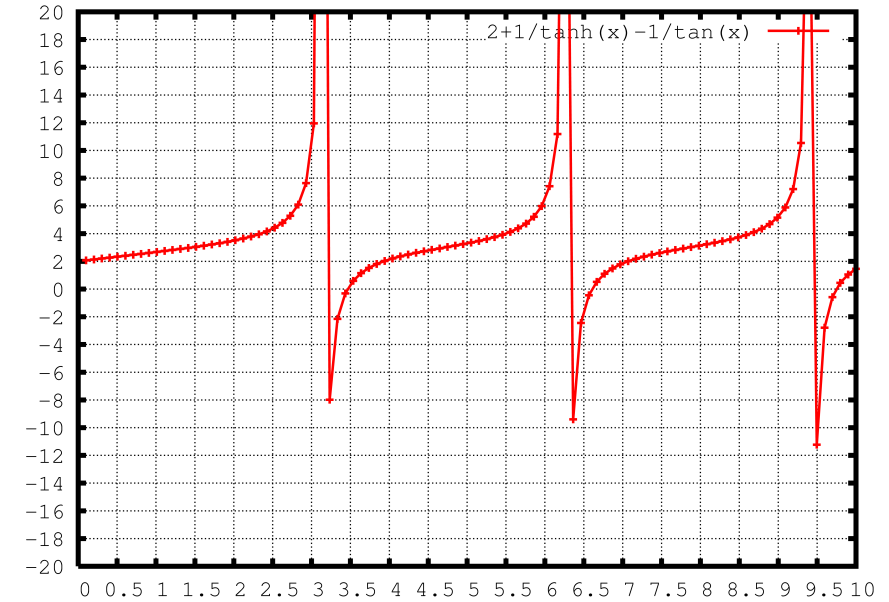

Fig. 3. The roots of this equation, $k_{n} L=3.4,6.6,9.75, \ldots$, are the frequencies of vibration of the $\alpha=1$ system.

Equation 2 is rearranged into

$$
\omega_{n}=k_{n}^{2} \sqrt{\frac{E I g}{A \gamma}}
$$

and $k$ is obtained as the solution of Eq. 15. The observable frequency, $\phi_{n}$ in Hertz, is simply the circular frequency divided by $2 \pi$ :

$$
\phi_{n}=\frac{k_{n}^{2}}{2 \pi} \sqrt{\frac{E I g}{A \gamma}}
$$

where $\phi_{n}$ represents the $n$th natural frequency of the beam-cable. It is seen that the frequency of vibration is inversely proportional to the square of the length of the beam, and proportional to the square root of the flexural rigidity.

The modal shapes are generated by the solution of Eq. 9:

$$
J=\frac{\sin k L}{\sinh k L} B
$$

so that, as depicted in Fig. 4 for the displacement and acceleration of the seventh natural frequency,

$$
X(x)=\sin k_{n} x+\frac{\sin k_{n} L}{\sinh k_{n} L} \sinh k_{n} x
$$

It is notable that, in Fig. 4, the first six half-waves have the period and amplitude of the sine wave, whereas the seventh and final half-wave shows a marked variation from the norm. The departure occurs at the wet end, the acceleration of which could be termed a whiplash..

\section{RESULTS}

The cable characteristics which were listed in Blanco, M. [2010] are repeated here in Table 2 and Fig. 5. The Milburn-Alembert analysis of VIV in Blanco, M. [2010],

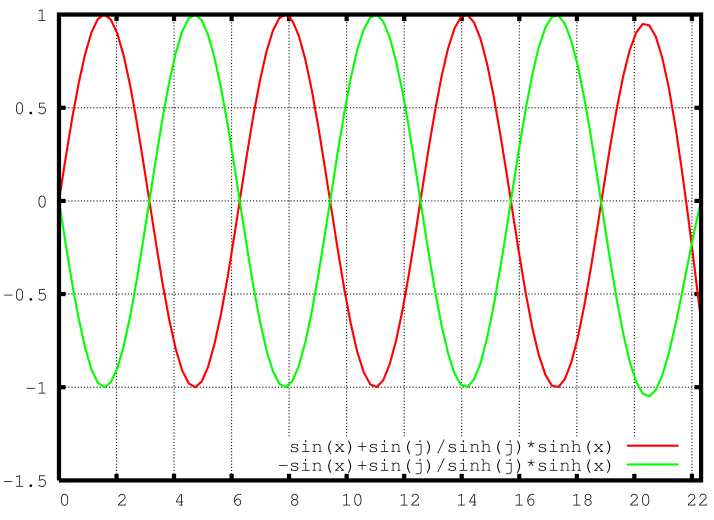

Fig. 4. Modal shape diagram for seventh natural frequency of beam-cable system.

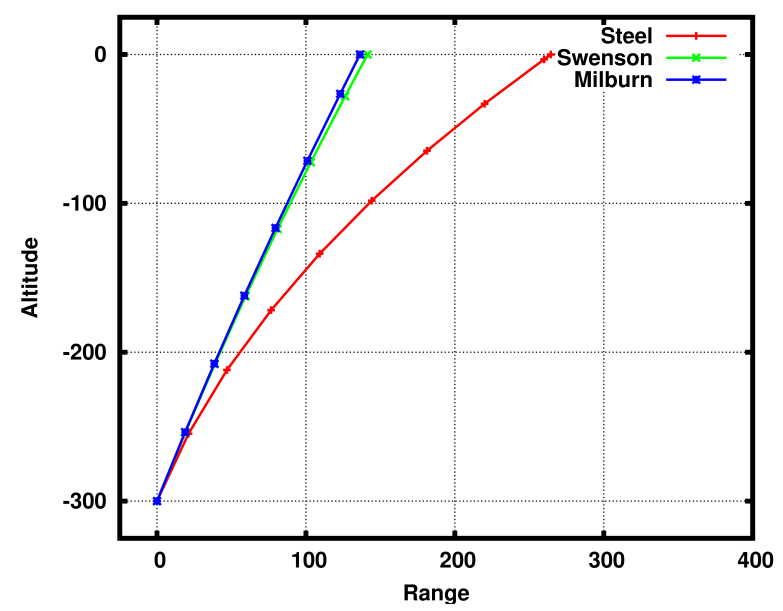

Fig. 5. Static cable analyses: range and depth for three cables.

which is repeated here in Table 3 , is suspect because of its coarse physics,

$$
f_{n}=\frac{n}{2 L} \sqrt{\frac{T}{m}}
$$

and is indicated by $f_{1}$ in Table 3 .

The first normal mode is recalculated here with the analysis of $\S 2.2$. Vibration modes can be said to be shaped by a ratio of 'inertial' force and 'restoration' force. The analysis of Milburn, D. A. [1983] can be said to have been overly simplistic, whereas Eq. 17 results from one that is more complex. The modal values obtained from the parameters of Table 2 are given in Table 5 .

The co-axial cable depicted in Fig. 6 is a simple model of the structure. Pesce, C. P., R. Ramos, Jr., L. M. Y. da Silveira, R. L. Tanaka, C. de Arruda Martins, F. C. M. Takafuji, J. P. Z. Novaes and C. A. F. Godinho [2010] is able to represent the various components of a modern umbilical cable. No effort has been been made to optimise the instant geometry, which is armoured by 24 concentric Kevlar tension members. 


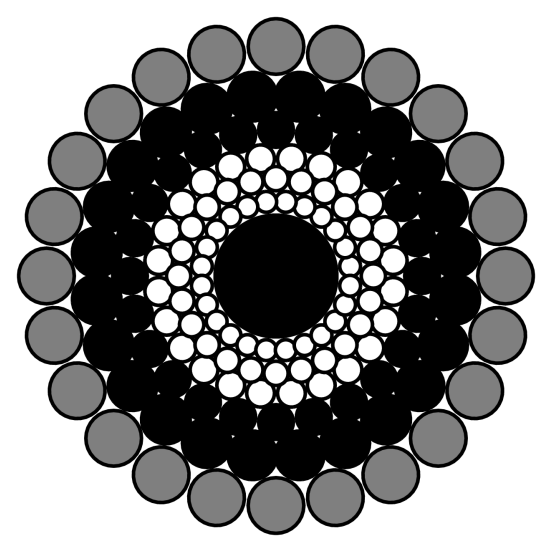

Fig. 6. Typical umbilical cable, sheathed with 24 strands of Kevlar (gray); center is high-voltage DC, while outer ring (in black) is ground potential. The conductors are separated by an insulator (white).

Since the core conductors and insulator are all at least an order of magnitude less stiff than the Kevlar tension members, they are treated as if they were transparent in the present study. In other words, the tension members are treated as a pipe or tube, and the moment of inertia is taken to be that of a disk about its central axis:

$$
\begin{aligned}
I_{o} & =\int_{A} y^{2} d A \\
& =\frac{\pi}{4}\left(r_{o}^{4}-r_{i}^{4}\right)
\end{aligned}
$$

It is important to differentiate between the 'second moment of inertia'" , denoted in $L^{4} F^{0} T^{0}$, and 'moment of inertia', denoted in $L^{1} F^{1} T^{2}$. The former quantity is employed for static problems like the present one, while the latter quantity is employed for problems of dynamics. No notice has been taken here of the 'virtual mass' effect in water, whereby the mass of the entrained fluid is calculated based on potential flow theory.

Table 4 lists the values employed in the calculation of Eq. 17. The result is $\phi_{1}=2.78 \times 10^{-3} \mathrm{~Hz}$, a similar order of magnitude as the Milburn model. Where the present model differs substantially from the Milburn taut string model is in the square of the wavenumber factor that appears in the former model. This implies that the smaller wavelength, higher wavenumber, modes are able to sustain audible frequencies. In concrete terms, the $300 \mathrm{ft}$ wavelength is unlikely to be excited, whereas the $1 \mathrm{ft}$ wavelength, wavenumber $k_{119}$ with frequency $70 \mathrm{~Hz}$, is likelier to be excited. Table 5 lists some wavenumbers and frequencies of vibration for this beam-cable system.

\section{CONCLUSION}

The sinusoidal function turns out to be the only trial solution for the transverse oscillations of an underwater 4 also known as the 'area moment of inertia' beam-cable system. The solution to the beam equation with pinned dry end and with a mass on the wet end was discovered and characterised. The modal shape was illustrated and can now be calculated.

The quantitative result for the first modal frequency of this particular beam-cable system is insignificant. The result corroborated that of Blanco, M. [2010], which employed a taut string model instead of the present beam-cable model. The present analysis differs in that the square of the wavenumber is a factor in the frequency equation, so that some in the audible range ought to manifest themselves. Experiments ought to be performed in order to verify these results.

\section{ACKNOWLEDGEMENTS}

This work, which was presented at the 8th IFAC Conference on Control Systems in Marine Applications, Rostock, 15-17 September 2010 while the first author was Marie Curie Research Fellow and Project Manager of $F R E E_{\text {sub }} N E T$, was supported by grant number MRTN036186 of the European Union Sixth Framework Project.

\section{REFERENCES}

Bishop, R. E. D., and D. C. Johnson. Mechanics of vibration. Cambridge University Press, Cambridge, 1960.

Blanco, M. Proposal for a shipborne AUV refuel system. Technical Report OMAE2010-20413, ASME Ocean, Offshore and Arctic Engineering Division, 2010. The 29th International Conference on Ocean, Offshore and Arctic Engineering Shanghai, China - June 6-11, 2010.

Han, S. M., H. Benaroya and T. Wei. Dynamics of transversely vibrating beams using four engineering theories. Journal of Sound and Vibration, 225(5):935988, 1999.

Laura, P. A. A., J. L. Pombo and E. A. Susemihl. A note on the vibrations of a clamped-free beam with a mass at the free end. Journal of Sound and Vibration, 37(2): 161-168, 1974.

Milburn, D. A. Hydrodynamic performance evaluation of a newly developed Kevlar rope fairing. In Proceedings 1983 Symposium on Buoy Technology, pages 25-31, Washington, DC, 1983. Marine Technology Society. New Orleans, Lousiana, April 27-29 1983.

Pesce, C. P., R. Ramos, Jr., L. M. Y. da Silveira, R. L. Tanaka, C. de Arruda Martins, F. C. M. Takafuji, J. P. Z. Novaes and C. A. F. Godinho. Structural behavior of umbilicals - part I: mathematical modelling. Technical Report 2010-20892, OMAE, 2010. 29th International Conference on Ocean, Offshore and Arctic Engineering, Shanghai, China 6-11 June 2010.

Prescott, J. Applied Elasticity. Dover Publications, Inc., New York, 1961.

Seto, W. W. Theory and Problems of Mechanical Vibrations. Schaum's Outline Series. McGraw-Hill Book Company, New York, 1964.

Timoshenko, S. and D. H. Young. Vibration problems in engineering. D. van Nostrand Company, Inc., London, 3rd edition, 1955. 


\begin{tabular}{lll} 
Mechanical Quantity & SI System & BGS System \\
\hline Linear velocity & $L M^{0} T^{-1}$ & $L F^{0} T^{-1}$ \\
Linear acceleration & $L M^{0} T^{-2}$ & $L F^{0} T^{-2}$ \\
Angular velocity & $L^{0} M^{0} T^{-1}$ & $L^{0} F^{0} T^{-1}$ \\
Angular acceleration & $L^{0} M^{0} T^{-2}$ & $L^{0} F^{0} T^{-2}$ \\
Mass & $L^{0} M T^{0}$ & $L^{-1} F T^{2}$ \\
Force & $L M T^{-2}$ & $L^{0} F T^{0}$ \\
Momentum, impulse & $L M T^{-1}$ & $L^{0} F T$ \\
Angular momentum & $L^{2} M T^{-1}$ & $L F T$ \\
Work, Energy, Torque & $L^{2} M T^{-2}$ & $L F T$ \\
Power & $L^{2} M T^{-3}$ & $L F T T^{-1}$ \\
Moment of inertia of mass & $L^{2} M T^{0}$ & $L F T^{2}$ \\
Moment of inertia of area & $L^{4} M M^{0}$ & $L^{4} F^{0} T^{0}$ \\
Flexural rigidity & & $L^{2} F^{1} T^{0}$
\end{tabular}

Table 1. Dimensions of the principal mechanical quantities.

Timoshenko, S., D. H. Young and W. Weaver, Jr. Vibration Problems in Engineering. John Wiley \& Sons, London, 4th edition, 1974.

Timoshenko, S. P. History of strength of materials: with a brief account of the history of theory of elasticity and theory of structure. McGraw-Hill, New York, 1953.

Volterra, E. and E. C. Zachmanoglou. Dynamics of vibrations. C. E. Merrill Books, Columbus, OH, 1965.

\section{NOMENCLATURE}

$\alpha \quad$ Mass ratio of plunger and beam-cable

$\gamma \quad$ (specific) weight per unit volume

$l b / i n^{3}$

$\omega_{n} \quad$ circular frequency of vibration

$\phi_{n} \quad$ frequency of vibration (beam-cable model)

$\rho$ mass density,

A cross-sectional area

E Young's modulus (of elasticity)

EI Flexural rigidity

$f_{n} \quad$ frequency of vibration (string model)

$g \quad$ acceleration due to gravity

I (area) moment of inertia of beam section

$k \quad$ wavenumber

$W_{p} \quad$ plunger weight

$x \quad$ Displacement along length of beam

$X(x) \quad$ Trial function

$1 / s$
$l b \cdot s^{2} / i n^{4}$
$i n^{2}$
$l b / i n^{2}$
$l b \cdot i n^{2}$
$1 / s$
$i n / s^{2}$
$i n^{4}$
$1 / i n$
$l b$
$i n$
$i n$

Table 1 lists the dimensions of the principal mechanical quantities in terms of length $L$, mass $M$, force $F$, and time $T$. This table serves as a check for dimensional congruity, without which the equation is said to be incorrect.

Equation 17 ought to result in $T^{-1}$; else it is incorrect:

$$
\phi_{n}=\frac{1}{L^{2} F^{0} T^{0}} \sqrt{\frac{L^{2} F^{1} T^{0} \cdot L^{1} F^{0} T^{-2}}{L^{2} F^{0} T^{0} \cdot L^{-3} F^{1} T^{0}}}
$$

Here, the radicand is comprised of the flexural rigidity and the acceleration due to gravity divided by the crosssectional area and the weight per unit volume.

\begin{tabular}{lrrr} 
Characteristic & Steel & Swenson & Milburn \\
\hline diameter, in & 0.35 & 0.43 & 0.30 \\
weight, lb $/ \mathrm{ft}$ & 0.15 & 0.02 & 0.01 \\
elasticity, $\times 10^{6} \mathrm{psi}$ & $9.0-$ & $9.0-$ & $9.0-$ \\
Reynolds number & 11,600 & 14,300 & 10,200
\end{tabular}

Table 2. Characteristics of three electromechanical cables - Imperial units.

\begin{tabular}{lccc} 
Variable & Steel & Swenson & Milburn \\
\hline$\phi$, in & 0.35 & 0.43 & 0.30 \\
$L, \mathrm{ft}$ & 405.6 & 331.8 & 329.6 \\
$T$ & 642.6 & 545.1 & 550.9 \\
$m, \mathrm{lb} / \mathrm{ft}$ & 0.15 & 0.02 & 0.01 \\
$S^{*}$ & 0.212 & 0.212 & 0.212 \\
$f^{*}, \mathrm{~Hz}$ & 49.0 & 39.9 & 57.2 \\
$f_{1}, \mathrm{~Hz}$ & 0.08 & 0.24 & 0.35 \\
$\mathrm{~N}$ & 612.5 & 166.25 & 163.42
\end{tabular}

Table 3. Blanco, M. [2010] calculations of the cable represented as a taut string.

\begin{tabular}{cccll} 
Variable & Value & Value & Units & Multiplier \\
\hline$E$ & 30. & 30. & $l b / \mathrm{in}^{2}$ & $\times 10^{6}$ \\
$I$ & 0.15 & 0.26 & $\mathrm{in} \mathrm{n}^{4}$ & 1 \\
$\gamma$ & 0.324 & 0.324 & $\mathrm{lb} / \mathrm{in}^{3}$ & 1 \\
$A$ & 0.58 & 0.58 & $\mathrm{in}$ & 1 \\
$L$ & 3960. & 3960. & $\mathrm{in}$ & 1 \\
$\phi_{1}$ & 2.78 &. & $1 / \mathrm{s}$ & $\times 10^{-3}$
\end{tabular}

Table 4. Values employed in calculations of $\phi_{n}$ for Swenson cable.

\begin{tabular}{rrrr}
$n$ & $k_{n} L$ & $k_{n}$ & $\phi_{n}$ \\
\hline 1 & 3.46 & 0.00087 & 0.0067 \\
2 & 6.60 & 0.00166 & 0.0247 \\
3 & 9.75 & 0.00246 & 0.0537 \\
4 & 12.88 & 0.00325 & 0.0938 \\
5 & 16.03 & 0.00405 & 0.1456 \\
6 & 19.17 & 0.00484 & 0.2080 \\
7 & 22.32 & 0.00564 & 0.2825 \\
8 & 25.45 & 0.00643 & 0.3671 \\
9 & 28.60 & 0.00722 & 0.4629 \\
10 & 31.75 & 0.00802 & 0.5711 \\
20 & 60.01 & 0.01515 & 2.0380 \\
40 & 119.70 & 0.03023 & 8.1145 \\
80 & 239.08 & 0.06037 & 32.3615 \\
119 & 352.19 & 0.08894 & 70.2393
\end{tabular}

Table 5. Values calculated for $\phi_{n}$ of Swenson cable. Units of $k_{n}$ are $1 /$ in, so $k_{119} \approx 1 \mathrm{ft}$. 


\title{
Autonomous Underwater Vehicle Pursuit of Biological Specimens in the Open Ocean
}

\author{
Max Blanco and Philip A. Wilson* \\ * School of Engineering Sciences, University of Southampton, \\ Southampton, UK, SO17 1BJ.
}

\begin{abstract}
The pursuit equations in two-dimensional space are examined, and then parametrised in terms of relative velocity and initial range. Several inferences about the behaviour of these equations are drawn. The burst speed of several fish species are tabulated, along with several types of Autonomous Underwater Vehicle. An example pursuit calculation is described.
\end{abstract}

Keywords: pursuit, fish, evasion, curves of pursuit, marine biology, autonomous underwater vehicle, AUV, strategy, analytic solution, path planning

\section{NOMENCLATURE}

$\lambda(f) \quad$ wavelength of sound frequency $f$ in water

a initial separation

c speed of sound in water

$c_{1} \quad$ constant of integration

$e \quad$ exponential function

$f \quad$ frequency of auditory signal

$k \quad$ speed of quarry

$n \quad$ ratio of pursuer speed to quarry speed

$P \quad$ location of AUV (pursuer)

$p \quad$ dummy variable for first derivative

$Q \quad$ location of fish (quarry)

$r \quad$ radius

$t \quad$ time

$v_{\max }$ burst speed

$x \quad$ first Cartesian coordinate

$y \quad$ second Cartesian coordinate

$y^{\prime} \quad$ first spatial derivative of $y$

$y^{\prime \prime} \quad$ second spatial derivative of $y$

\section{INTRODUCTION}

Some population studies in marine biology require that fish be tracked. This need is exemplified by a research partner, the University of the Azores, whose biologists seek to locate particular specimens which have been tagged with acoustic emitters. The biologists hope the acoustic signals which are emitted can indicate the habitat and/or behaviour of the specimen, shoal, or species. The symbiotic relationship between the present researchers and the biologists is to provide the latter with Autonomous Underwater Vehicle (AUV) tools to achieve their goals. Progress towards the shared goal will be obtained if the mathematics of biological pursuit is clarified. Properly defined, the set of all pursuits includes the stationary quarry.

This paper will be organised as follows: the next section will consist of a literature survey. Curves of pursuit will be explained in $\S 3$. Some biological applications of the mathematical tool will be explored in consequence. Recommendations for further study will complete the paper.

\section{LITERATURE STUDY}

The differential equations of pursuit were developed as a result of World War II, so that Yates, R. C. [1952] §9.5.G devoted scarcely two pages to treatment of the subject. These equations are repeated and developed here in the next section. Standard reference material even today was written by Locke, A. S. [1955]. Section 7.9 of Langer, R. E. [1954] had the pursuer and quarry in opposite corners of the Cartesian system, but his results were similar to those of Yates. Stewart did not teach the problem in his first edition Stewart, J. [1987] but added a treatment similar to that of Langer in his third Stewart, J. [2006] at page 554.

The example to be employed in the next section follows Yates, R. C. [1952] closely because his placement of the origin coincides with the initial location of the pursuer. The terminal $v^{2} / r$ acceleration problem of military pursuit is of no concern because, as explained by Fig. 1, the pursuit of a specimen differs from its capture. The biologists forbid specimen capture in the present instance. Adler, F. P. [1955] knew of three types of guidance systems: pure pursuit, constant-bearing collision, and proportional navigation. His theory of proportional navigation, as updated with complex coordinates by Becker, K. [1990], is still relevant today in the case of the marine biologist. His reason for the elimination of constant-bearing collisionthat the algorithm requires instantaneous adjustments of the line-of-sight-holds true for the present application too. He eliminated for his purposes the first option on two grounds: (a) that the mathematics of the pursuit equations forces the system into a tail-chase scenario (which would be unsuitable for miltary defense); and (b) that the terminal turn rate becomes infinite if the ratio of pursuer to quarry speed exceeds two. Doppler effect logic can be 


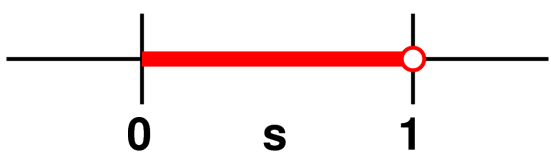

Fig. 1. The strict definition of the problem includes pursuit, marked in red on this numberline, but not capture, as evinced by the domain termination at $s \in[0,1)$.

employed for biological purposes so that the AUV is only allowed to engage its quarry while the quarry recedes from it, while inspection of Fig. 3 shows his error in case (b) to be of similar type with Zeno's paradox. This study appears to be novel since his ground (and those of his peers) differs from the present ground.

The case of curvature constrained pursuit is of interest, because curvature is the mirror image of centripetal acceleration. Miloh, T. [1982] notes that in certain instances it can be advantageous to both parties to maintain a planar relationship in three-dimensional space, and that the termination of his game-theoretic approach depends substantially on the maximum rate of acceleration of both parties. While the minimum rate of turn of the quarry is unknown and unknowable, the minimum rate of turn of the pursuer is knowable. More recent efforts in this area have focussed on collision avoidance Harris, C. J., et al. [1999], Wilson, P. A., C. J. Harris, X. Hong [2003], or incomplete measurements and uncertain systems, eg. Moitié, R., et al. [2002] or Shieh, C.-S. [2007]. The latter can be of very limited tractability due to memory requirements Moitié, R., et al. [2002] or are tunable Shieh, C.-S. [2007], thus unsuited to automation. But the presumption of the latter that the object of the mathematics is to destroy the quarry is contrary to expectations in the present study. The marine biologist whose aim is observation of the quarry at close range is likely to avoid damage to it, and hence the minimum turn radius is likely to be irrelevant, whereas the military objective, which has informed most previous studies of pursuit, is destruction. Figure 3 demonstrates the need to avoid velocity ratios near to unity because this causes more curvature to be required in the path to intercept.

\section{THEORY}

The Yates exposition of the pursuit equation, which is repeated here in Fig. 2, differs from the Langer presentation of the same problem in its choice of origin: Yates favours the pursuer, while Langer favours the quarry. The AUV (pursuer, $P$ ) and the specimen (quarry, $Q$ ) are labelled. The quarry travels in a straight line at maximum speed, while the pursuer acts at all times to minimise its distance to the quarry; that is, $P$ makes no effort to predict the behaviour of $Q$, and hence it is at all times directed towards $Q$. The quarry travels Northward at maximum speed $k$. The pursuer notices $Q$ at time $t_{o}$ near $O$, the

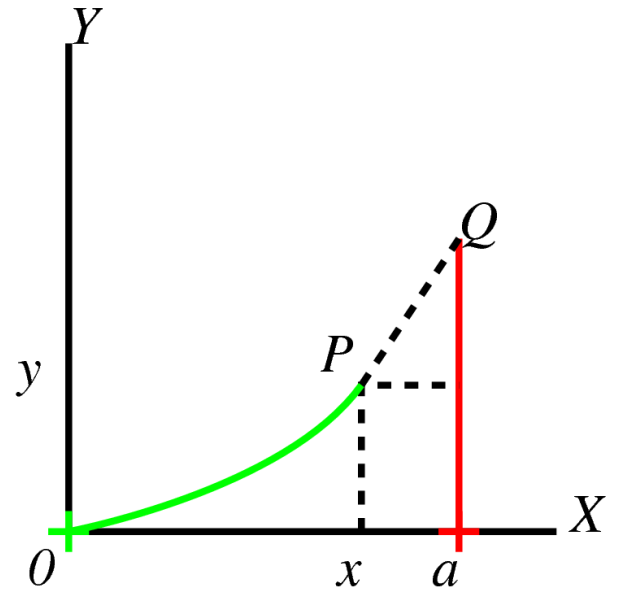

Fig. 2. The problem from the viewpoint of the pursuer, whose path is marked in green. The path of the quarry is marked in red. A cross marks the origin of each.

origin, which is a metres distant from the instant location of $Q$. Suppose $n k$ to be the speed of $P$, and $r$ to be the range between the two:

$$
\begin{aligned}
n k & =\frac{d r}{d t} \\
& =\frac{\sqrt{d x^{2}+d y^{2}}}{d t} \\
& =\frac{d x \sqrt{1+y^{\prime 2}}}{d t}
\end{aligned}
$$

and the stem of $P$ to be pointed at the location

$$
\frac{d y}{d x}=y^{\prime}=\frac{k t-y}{a-x}
$$

Equation 4 completes the physics of the problem, and the mathematics now follows:

$$
\begin{aligned}
y^{\prime}(a-x) & =k t-y \\
y^{\prime \prime}(a-x)-y^{\prime} & =\quad k \frac{d t}{d x}-y^{\prime} \\
y^{\prime \prime}(a-x)-y^{\prime} & =k \frac{\sqrt{1+y^{\prime 2}}}{n k}-y^{\prime} \\
n y^{\prime \prime}(a-x) & =\sqrt{1+y^{\prime 2}}
\end{aligned}
$$

The result is a nonlinear equation of the second order in which the term $y$ makes no explicit appearance. This allows the substitution $y^{\prime}=p$ to transform the equation for the path of $P$ to

$$
\begin{gathered}
n(a-x) \frac{d p}{d x}=\sqrt{1+p^{2}} \\
\frac{n d p}{\sqrt{1+p^{2}}}=\frac{d x}{a-x}
\end{gathered}
$$


a directly-integrable form which has the general solution

$$
n \ln \left(p+\sqrt{1+p^{2}}\right)=\ln \left(\frac{c_{1}}{a-x}\right)
$$

Notice is taken of the initial condition that

$$
\left.p\right|_{x=0}=\left.y^{\prime}\right|_{x=0}=0
$$

determines $c_{1}=a$, and so

$$
\begin{aligned}
n \ln \left(p+\sqrt{1+p^{2}}\right) & =\ln \left(\frac{a}{a-x}\right) \\
\ln \left(p+\sqrt{1+p^{2}}\right) & =\ln \left(\frac{a}{a-x}\right)^{1 / n}
\end{aligned}
$$

The left-hand side is shown by Stewart, J. [1987] in his Eq. 6.80 to be amenable to trigonometric substitution:

$$
\ln \left(p+\sqrt{1+p^{2}}\right)=\sinh ^{-1} p
$$

and so

$$
\begin{array}{r}
p=\sinh \left[\ln \left(\frac{a}{a-x}\right)^{1 / n}\right] \\
\frac{d y}{d x}=\sinh \left[\ln \left(\frac{a}{a-x}\right)^{1 / n}\right]
\end{array}
$$

but Stewart, J. [1987] defines in his Eq. 6.76,

$$
\sinh \phi=\frac{e^{\phi}-e^{-\phi}}{2}
$$

Now since

$$
\exp \left[\ln \left(\frac{a}{a-x}\right)^{1 / n}\right]=\left(\frac{a}{a-x}\right)^{1 / n}
$$

and

$$
\begin{gathered}
\exp \left[-\ln \left(\frac{a}{a-x}\right)^{1 / n}\right]=\exp \left[\ln \left(\frac{a}{a-x}\right)^{-1 / n}\right] \\
=\left(\frac{a}{a-x}\right)^{-1 / n}
\end{gathered}
$$

the result is

$$
\begin{gathered}
2 \frac{d y}{d x} \quad=\left(\frac{a}{a-x}\right)^{1 / n}-\left(\frac{a}{a-x}\right)^{-1 / n} \\
2 d y=\left[a^{1 / n}(a-x)^{-1 / n}-a^{-1 / n}(a-x)^{1 / n}\right] d x \\
2 y=\frac{a^{1 / n}(a-x)^{1-1 / n}}{1-1 / n}+\frac{a^{-1 / n}(a-x)^{1+1 / n}}{1+1 / n} \\
-\frac{2 n a}{1-n^{2}}
\end{gathered}
$$

where the constant of integration

$$
\frac{-2 n a}{1-n^{2}}
$$

is obtained from the observation that $y=0$ when $x=0$.

\begin{tabular}{lll} 
Species & $\bar{v}$ & $v_{\max }$ \\
\hline Alosa pseudoharengus & & 4.7 \\
Clupea harengus & & 1.7 \\
Esox lucius & 1.4 & 2.0 \\
Gadus morhua & 0.5 & \\
Micropterus salmoides & 0.9 & \\
Oncorhynchus gorbuscha & 0.6 & \\
Pleuronectes platessa & 0.3 & \\
Sarda sarda & 0.3 & 1.2 \\
Sphyraena barracuda & & 12.1 \\
Scomber scombrus & 0.9 & 3.0 \\
Thunnus albacares & 0.6 & 12.4 \\
\hline Homo sapiens WR & 1.7 & 2.1
\end{tabular}

Table 1 . The average speed and burst speed, measured in $\mathrm{m} / \mathrm{s}$, of various fish species as compiled by Froese, R. and D. Pauly [2010], contrasted with freestyle swim world records for $1500 \mathrm{~m}$ and $100 \mathrm{~m}$ events.

\begin{tabular}{llllll} 
Species & $v_{\max }$ & Depth & Weight & Length & Diameter \\
\hline Bluefin 9 & 2.6 & 100 & 50 & 1.65 & 9 \\
Remus 100 & 2.8 & 100 & 37 & 1.60 & 7.5 \\
Gavia & 2.8 & 500 & 50 & 1.80 & 7.9
\end{tabular}

Table 2. Statistics on three 'man-portable'

AUVs. All dimensions are compiled in SI units except inches diameter.

\section{RESULTS}

Equation 26 has been parametrised as a function of $a$ and $n$, which are respectively: the initial separation and the relative ratio of pursuit to quarry velocities. This is depicted in Fig. 3 for three velocity ratios, $n=4,2,5 / 4$. Several characteristics of the family of curves can be seen immediately:

- The pursuer never reaches the quarry if $n=1$; so a simple evasion tactic is to match or exceed the speed of the pursuer.

- A corollary is that the pursuer does not engage if $n \leq 1$.

- The interval between engagement and encounter is calculable a priori when the pursuit is engaged.

- The location of encounter is known when the pursuit is engaged.

A successful collaboration between biologists and engineers thus requires that the speed of the fish be among the available data.

The behaviour of fish is studied by marine biologists such as Korsmeyer, K. E., et al. [2002] and Cartamil, D. P., et al. [2003]; the velocity of travel of the specimens is but one of the measures. Table 1 presents these statistics, as well as some gathered from Froese, R. and D. Pauly [2010]. These statistics are to be compared with the list of 'portable' AUVs in Table 2. The majority of fish species identified in this paper are pursuable by small AUVs of the types listed here. The larger predatory fish like the barracuda and the tuna would require greater speeds of pursuit. 
The 'Remus 100' class of AUVs, which has a maximum velocity of $2.8 \mathrm{~m} / \mathrm{s}$, is able to catch Sarda sarda ${ }^{1}$, which has a burst speed of $1.2 \mathrm{~m} / \mathrm{s}$, because the velocity ratio $n=2.8 / 1.2=7 / 3$ is greater than unity. This AUV will require $437.5 \mathrm{~s}$ (7.5 minutes) to intercept its quarry when initially they are separated by $1000 \mathrm{~m}$, if indeed the bonito can sustain its burst speed over that interval.

In practice, the algorithm would have a 'stand-off' distance to protect the specimen from interaction with it. This distance would prevent collision between the two or exhaustion of the specimen. This accords well with the exclusion of capture specified by Fig. 1 .

Marine acoustic technology has developed enough to allow sound navigation and ranging to achieve a high level of accuracy for sophisticated users. Signals must be received by at least two devices in order to detect target bearing. It is unknown whether present AUV technology supports stereophonic reception. Table 2 suggests that a stereo receiver array might be separated by at most one metre. Commercially-available transmitters are available which broadcast at $f=69 \mathrm{kHz}$. The speed of sound in water is roughly $c=1500 \mathrm{~m} / \mathrm{s}$, so the wavelength of $\lambda(69 \mathrm{kHz})=$ $c / f \approx 0.02 \mathrm{~m}$ is in theory resoluble.

\section{CONCLUSION}

The evasion strategy of common fish is unknown but knowable; further biological research to describe the behaviour of these animals is needed. Focus on the minimum radius of turn under evasive circumstances might prove beneficial. The 'man-portable' AUVs listed in this study are able in theory to intercept low-speed tagged fish. Contemporary technology seems to be unable to offer an AUV which is able to intercept such high-speed fish as the barracuda.

AUVs are notorious for their power and endurance limitations; in an imagined future, the AUV might query various speeds for their effect on intercept time and power consumption, and decide on its optimum action.

This paper provides the mathematical solution to the path of pursuit in the open ocean given the observables target range, target speed, and target course or bearing. The AUV is fed this data to pursue the target. The open ocean is however three-, not two-dimensional as was the simplified case above. Three-dimensional solutions to the equations of pursuit have been reviewed in gametheoretic terms by Miloh, T. [1982], who states that under certain conditions both pursuer and quarry find the planar solution to be optimal. The instant solution is therefore applicable to the concrete world, however the success of the algorithm described here remains to be seen in reality.

\section{ACKNOWLEDGEMENTS}

This work was supported by grant number MRTN-036186 of the European Union Sixth Framework Project. Presented at the 8th IFAC Conference on Control Systems in Marine Applications, Rostock, 15-17 September 2010, while the first author was Marie Curie Research Fellow and Project Manager of $F R E E_{\mathrm{Sub}} N E T$. The first author is indebted for remarks on $v^{2} / r$ acceleration to an anonymous reviewer, and to Mara Schmiing of IMAR for her original scenario and bibliography on marine biology.

\section{REFERENCES}

Adler, F. P. Missile guidance by three-dimensional proportional navigation. Journal of Applied Physics, 27: 500-507, 1955.

Becker, K. Closed-form solution of pur proportional navigation. IEEE Transactions on Aerospace and Electronic Systems, 26(3):526-533, May 1990.

Cartamil D. P., J. J. Vaudo, C. G. Lowe, B. M. Wetherbee and K. N. Holland. Diel movement patterns of the hawaiian stingray, dasyatis lata: implications for ecological interactions between sympatric elasmobranch species. Marine Biology, 142(5):841-847, 2003.

Froese, R. and D. Pauly. Speed Measurements, 2010. Database accessed 31 March 2010 at www . fishbase. org/Topic/List . php?group $=32$.

Harris, C. J., X. Hong, P. A. Wilson. An intelligent guidance and control system for ship obstacle avoidance. Proc. IMechE, Part I: Journal of Systems and Control Engineering, 213(4):311-320, 1999.

Korsmeyer, K. E., J. F. Steffensen, J. Herskin. Energetics of median and paired fin swimming, body and caudal fin swimming, and gait transition in parrotfish (scarus schlegeli) and triggerfish (rhinecanthus aculeatus). The Journal of Experimental Biology, 205:1253-1263, 2002.

Langer, R. E. A first course in ordinary differential equations. John Wiley \& Sons, London, 1954.

Locke, A. S. Guidance. D. Van Nostrand Company, Inc., London, 1955.

Miloh, T. A note on three-dimensional pursuit-evasion game with bounded curvature. IEEE Transactions on Automatic Control, AC-27(3):739-741, June 1982.

Moitié, R., M. Quincampoix and V. M. Veliov. Optimal control of discrete-time uncertain systems with imperfect measurement. IEEE Transactions on Automatic Control, 47(11):1909-1914, November 2002.

Shieh, C.-S. Design of three-dimensional missile guidance law via tunable $\mathrm{h}_{\infty}$ control with saturation constraint. IET Control Theory Applications, 1(3):756-763, May 2007.

Stewart, J. Calculus. Thomson Brooks/Cole, Belmont, CA, 3rd edition, 2006.

Stewart, J. Calculus. Brooks/Cole Publishing Company, Pacific Grove, CA, 1987.

Wilson, P. A., C. J. Harris, X. Hong. A line of sight counteraction navigation algorithm for ship encounter collision avoidance. Journal of Navigation, 56(1):111121, 2003.

Yates, R. C. Differential equations. McGraw-Hill Book Company, Inc., London, 1952.

\footnotetext{
1 commonly known as the Atlantic bonito
} 


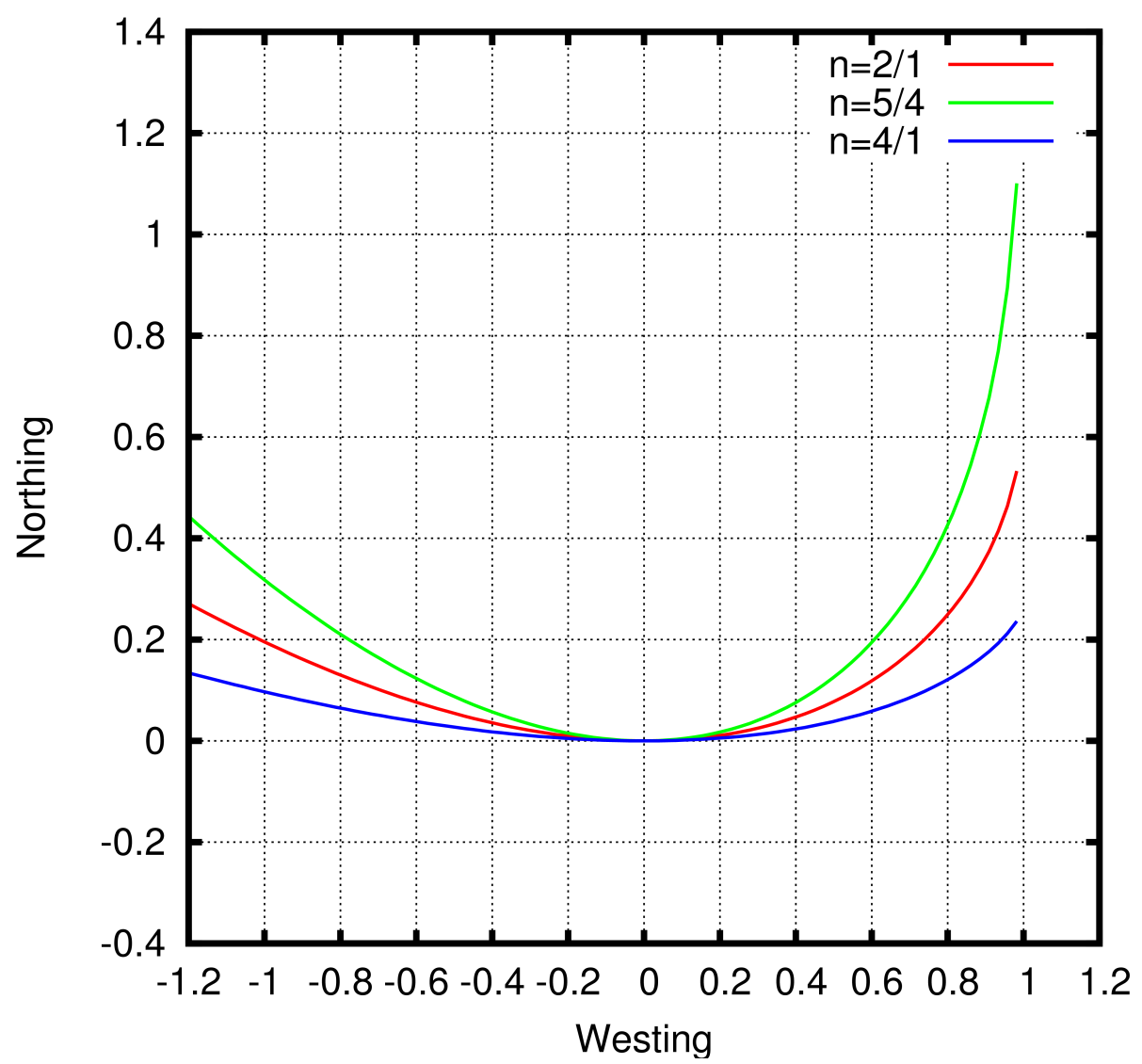

Fig. 3. Three curves of pursuit: $n=5 / 4,2,4$. The domain and range are both in quadrant I. 


\title{
Autonomous Underwater Vehicle Minimum-Time Navigation in a Current Field
}

\author{
Max Blanco and Philip A. Wilson \\ Fluid-Structure Interactions Group \\ School of Engineering Sciences \\ University of Southampton \\ Southampton, UK \\ e: m.blanco@soton.ac.uk
}

\begin{abstract}
The problem of navigation in a spatially variable current is reviewed, and for a certain class of mathematicallydescribable functions, solved for minimum time in closed form.

\section{NOMENCLATURE}

$\dot{\theta} \quad$ rate of change of heading (angular velocity)

$\dot{x} \quad x$-directed component of vehicle velocity

$\dot{y} \quad y$-directed component of vehicle velocity

$F \quad n$-dimensional constraint vector

$\lambda \quad n$ Lagrange multipliers

$\theta \quad$ vehicle course or heading (control input)

$U \quad m$-dimensional control input, decision vector

$\vec{i} \quad$ unit vector

$\overrightarrow{\mathrm{j}} \quad$ unit vector

$\vec{c} \quad$ velocity of current

$X \quad n$-dimensional state vector

$g(x, y) x$-directed component of current

$h(x, y) y$-directed component of current

$J \quad$ a scalar functional

$L \quad$ performance index

$m$ number of control inputs

$n$ number of components of state vector

$r \quad$ representative length

$V \quad$ vehicle velocity relative to the water

$x \quad$ Cartesian component (abscissa)

$y \quad$ Cartesian component (ordinate)

$x \in X$

$y \in X$
\end{abstract}

\section{INTRODUCTION}

To optimise navigation with current in two-dimensional space is known as Zermelo's problem, from [5]. The ability to compensate for current is a vital tool for Autonomous Underwater Vehicles (AUVs). Waypoints, docks and other quasistationary targets need to be reached by the AUV in minimum time, if its range is to be maximised under conditions of constant velocity. The solution to this problem is available under certain conditions in closed form, which allows the validation of algorithms designed to govern the navigation of these vehicles. One alternate means to specify the problem that will not be investigated here is that the energy consumed by the AUV be minimised over the trajectory from $A$ to $B$. Another unexplored option in the same current field is to control the velocity in order to ensure a straight-line course.
This last option is not recommended for practical applications because under some circumstances the current may exceed the maximum velocity of the vehicle.

Zermelo's problem is solved optimally here by means of the Pontryagin maximum principle [2] with the methodology of Bryson and Ho [1, §2.7]. Extensions to this class of problem were published by Zlobec [6]. The paper is subdivided into an abstraction, an analysis, and the case of linear current distribution is solved.

Smith et al. [3] have recently investigated the use of the three-dimensional current predictions of the JPL OurOcean portal and found that an unscented Kalman filter (UKF) algorithm seems reliable to predict vehicle paths over a $2 \mathrm{~km}$ range. The $2 \mathrm{~km}$ range is here taken as a representative measure; the AUV is tasked with minimum time rendez-vous with the waypoint.

\section{Abstraction}

The functional that is minimised here is just

$$
J=\int_{t_{A}}^{t_{B}} L(X(t), U(t)) d t
$$

which must be a stationary point in the two hyperplanes, $X$ and $U$, of the Hamiltonian, $\varkappa$ :

$$
\delta J=\frac{\partial \varkappa}{\partial U} \delta U+\frac{\partial \varkappa}{\partial X} \delta X
$$

The vector $X$ represents the state (position) vector, while $U$ represents the control input vector. The function $L(X, U) \equiv 1$, which implies

$$
J=t_{B}-t_{A}
$$

and allows the statement of a minimum-time navigation problem, subject to constraints

$$
\dot{X} \equiv \frac{d X}{d t}=F(X, U)
$$

where the overdot notation is employed to indicate the time derivative, and optimality conditions

$$
0=F_{u}^{T} \lambda
$$

obtain. Subscripts, in general, denote differentiation. Here, the Hamiltonian

$$
\varkappa=L+F_{u}^{T} \lambda
$$


is comprised of the performance index, $L$, and the Lagrange multipliers, $\lambda$, compounded by the constraint functions, $F$, transposed. This abstraction maps onto the navigation problem in what follows with $[x, y] \in X$ and $[\theta] \in U$. That is, the state vector is identical to the position of the vehicle, while the heading angle is the only control variable.

\section{AnAlysis}

The current field is of known magnitude and direction,

$$
\vec{c}=g(x, y) \vec{\imath}+h(x, y) \vec{\jmath}
$$

in the Cartesian plane. Vehicle velocity relative to the water is $V$, and constant in magnitude, and as a result the equations of motion can be written

$$
\begin{aligned}
& \dot{x}=V \cos \theta+g(x, y) \\
& \dot{y}=V \sin \theta+h(x, y)
\end{aligned}
$$

where overdot notation is written for the time derivative and $\theta$ represents the vehicle course with respect to an earth-fixed orthogonal coordinate frame. The Hamiltonian of the system is

$$
\varkappa=\lambda_{x}(V \cos \theta+g)+\lambda_{y}(V \sin \theta+h)+1
$$

so

$$
\begin{aligned}
\dot{\lambda_{x}} & =-\frac{\partial \varkappa}{\partial x}=-\lambda_{x} \frac{g}{x}-\lambda_{y} \frac{h}{x} \\
\dot{\lambda_{y}} & =-\frac{\partial \varkappa}{\partial y}=-\lambda_{x} \frac{g}{y}-\lambda_{y} \frac{h}{y} \\
0 & =\frac{\partial \varkappa}{\partial \theta}=V\left(-\lambda_{x} \sin \theta+\lambda_{y} \cos \theta\right)
\end{aligned}
$$

where Eq. 13 implies that

$$
\tan \theta=\frac{\lambda_{y}}{\lambda_{x}}
$$

The Hamiltonian is time invariant. Moreover, it equates to zero because an extremal (minimum-time) solution is desired. The system of equations 11 and 12 is solved ${ }^{1}$ for $\lambda_{x}$ and $\lambda_{y}$ :

$$
\begin{aligned}
\lambda_{x}(V \cos \theta+g)+\lambda_{y}(V \sin \theta+h) & =-1 \\
\lambda_{x}(-V \sin \theta)+\lambda_{y}(V \cos \theta) & =0
\end{aligned}
$$

to obtain

$$
\begin{aligned}
& \lambda_{x}=\frac{-\cos \theta}{V+g \cos \theta+h \sin \theta} \\
& \lambda_{y}=\frac{-\sin \theta}{V+g \cos \theta+h \sin \theta}
\end{aligned}
$$

and an equation for the rate of change of heading angle is the result:

$$
\dot{\theta}=\sin ^{2} \theta \frac{\partial h}{\partial x}+\sin \theta \cos \theta\left(\frac{\partial u}{\partial x}-\frac{\partial h}{\partial y}\right)-\cos ^{2} \theta \frac{\partial g}{\partial y}
$$

The three rate equations, Eqs. 8, 9 and 19, will determine the minimum-time paths through a terminal point $B$ when the initial coordinates $A$ and course, $\theta_{A}$, are set. What follows will place the destination at the origin $(0,0)$ of the coordinate axes, and the initial point $A$ somewhere in the domain.

\footnotetext{
${ }^{1}$ see Appendix
}

\section{Linear CurRent Distribution}

The case of a linear current distribution, $g=-V / r y, h=0$ is addressed here. This current is irrotational and meant to model a shear flow in $y$. The terminal heading angle $\theta_{B}$ is assumed to be known and collinear with the terminal velocity.

$$
\begin{aligned}
\cos \theta & =\frac{\cos \theta_{B}}{1+y / r \cos \theta_{B}} \\
\cos \theta+\frac{y}{r} \cos \theta_{B} \cos \theta & =\cos \theta_{B} \\
\frac{y}{r} \cos \theta_{B} \cos \theta & =\cos \theta_{B}-\cos \theta \\
\frac{y}{r} & =\frac{1}{\cos \theta}-\frac{1}{\cos \theta_{B}} \\
\frac{y}{r} & =\sec \theta-\sec \theta_{B}
\end{aligned}
$$

The rate equation for $\theta$ is solved next:

$$
\begin{aligned}
\dot{\theta}= & \sin ^{2} \theta \frac{\partial h}{\partial x}-\cos ^{2} \theta \frac{\partial g}{\partial y}+ \\
& \sin \theta \cos \theta\left(\frac{\partial g}{\partial x}-\frac{\partial h}{\partial y}\right) \\
\dot{\theta}= & -\cos ^{2} \theta \frac{\partial g}{\partial y} \\
\frac{d \theta}{d t}= & \cos ^{2} \theta \frac{V}{r} \\
\frac{d t}{d \theta}= & \frac{r}{V} \sec ^{2} \theta \\
\int_{A}^{B} \frac{V}{r} d t= & \int_{\theta_{A}}^{\theta_{B}} \sec ^{2} \theta d \theta \\
\frac{V}{r}\left(t_{B}-t_{A}\right)= & \tan \theta-\tan \theta_{B}
\end{aligned}
$$

Equation 31, which encodes the functional $J$ from Eq. 3, is also known as the performance index.

The rate equation for $x$ is solved last:

$$
\begin{aligned}
\frac{d x}{d t} & =V \cos \theta+g \\
& =V \cos \theta-V \frac{y}{r} \\
\frac{d x}{d \theta} \frac{d \theta}{d t} & =V \cos \theta-V \sec \theta+V \sec \theta_{B} \\
\frac{d x}{d \theta} & =\left[V \cos \theta-V \sec \theta+V \sec \theta_{B}\right] \frac{d t}{d \theta} \\
\frac{d x}{d \theta} & =\left[V \cos \theta-V \sec \theta+V \sec \theta_{B}\right] \frac{r}{V} \sec ^{2} \theta \\
\frac{d x}{d \theta} & =\frac{V \cos \theta-V \sec \theta+V \sec \theta_{B}}{V / r \cos ^{2} \theta} \\
d x & =\left[r \sec \theta-r \sec { }^{3} \theta+r \sec \theta_{B} \sec ^{2} \theta\right] d \theta \\
\frac{x}{r} & =\int_{\theta_{B}}^{\theta_{A}}\left[\sec \theta-\sec { }^{3} \theta+\sec \theta_{B} \sec ^{2} \theta\right] d \theta \\
& =-\frac{1}{2}\left[\sec \theta_{B}\left(\tan \theta_{B}-\tan \theta_{A}\right)-\right. \\
\log \frac{\tan \theta_{B}+\sec \theta_{B}}{\tan \theta_{A}+\sec \theta_{A}}- & \left.\tan \theta_{A}\left(\sec \theta_{B}-\sec \theta_{A}\right)\right]
\end{aligned}
$$




\section{RESUlTS}

Equations 24 and 40 constitute an implicit system for $\theta_{A}$ and $\theta_{B}$, and are solved by numerical methods. Suppose an AUV desires to travel from $\left(x_{A} / r, y_{A} / r\right)=(3.66,-1.86)$ to the origin, then

$$
\begin{array}{r}
\frac{y_{A}}{r}=-1.86=\sec \theta_{A}-\sec \theta_{B} \\
\frac{x_{A}}{r}=3.66=-\frac{1}{2}\left[\sec \theta_{B}\left(\tan \theta_{B}-\tan \theta_{A}\right)-\right. \\
\log \frac{\tan \theta_{B}+\sec \theta_{B}}{\tan \theta_{A}+\sec \theta_{A}}- \\
\left.\tan \theta_{A}\left(\sec \theta_{B}-\sec \theta_{A}\right)\right]
\end{array}
$$

This system of equations is intractable because it is composed of the trigonometric tangent and secant functions, both of which are in places unbounded. Equations 41 and 42 are modified to equate to zero, and the map of the $L_{2}$-norm is derived over a Cartesian solution plane composed of $\theta_{A}$ and $\theta_{B}$. The map is generated on every odd-numbered degree in order to avoid the infinities, and then scrutinised in regions which contain small values. The algorithm, although robust, can be said to be in want of refinement. The solution, which requires at most 32,400 iterations of two cosines, two tangents, one logarithm and one square root function, is found to be for this example,

$$
\theta_{A}=105^{\circ} \quad \theta_{B}=240^{\circ}
$$

\section{CONCLUSions AND Future Work}

Although one is unlikely to meet a well-ordered linear shear current in nature, the present result can be employed off-line to validate algorithms for navigation in a current. The abstraction needs to be automated in order to introduce the ability to navigate in regions with arbitrary current distributions.

\section{ACKNOWLEDGEMENTS}

This research was performed while the first author was employed under contract number MRTN-2006-036186 of the Sixth Framework Project of the European Commission.

\section{APPENDIX}

\section{A. Isolation of $\lambda_{x}, \lambda_{y}$}

The system of equations is solved as follows. Isolate from Eq. $16 \lambda_{x}$

$$
\begin{aligned}
\lambda_{x}= & \frac{-V \cos \theta}{-V \sin \theta} \lambda_{y} \\
& =\frac{\cos \theta}{\sin \theta} \lambda_{y}
\end{aligned}
$$

and substitute the result into Eq. 15:

$$
\begin{aligned}
-1 & =\lambda_{y}\left(\frac{\cos \theta}{\sin \theta}\right)[V \cos \theta+g]+\lambda_{y}[V \sin \theta+h] \\
-\sin \theta & =\lambda_{y} \cos \theta[V \cos \theta+g]+\lambda_{y}\left[V \sin ^{2} \theta+h \sin \theta\right] \\
-\sin \theta & =\lambda_{y}\left[V \cos ^{2} \theta+g \cos \theta+V \sin ^{2} \theta+h \sin \theta\right] \\
-\sin \theta & =\lambda_{y}[V+g \cos \theta+h \sin \theta] \\
\lambda_{y} & =\frac{-\sin \theta}{V+g \cos \theta+h \sin \theta}
\end{aligned}
$$

Isolate from Eq. $16 \lambda_{y}$

$$
\lambda_{y}=\lambda_{x} \frac{\sin \theta}{\cos \theta}
$$

and substitute the result into Eq. 15:

$$
\begin{aligned}
0 & =\lambda_{x}[V \cos \theta+g]+\lambda_{x} \frac{\sin \theta}{\cos \theta}[V \sin \theta+h]+1 \\
-1 & =\lambda_{x}\left[\frac{V \cos \theta^{2}+g \cos \theta+V \sin \theta^{2}+h \sin \theta}{\cos \theta}\right] \\
-1 & =\lambda_{x}\left[\frac{V+g \sin \theta+h \sin \theta}{\cos \theta}\right] \\
\lambda_{x} & =\frac{-\cos \theta}{V+g \cos \theta+h \sin \theta}
\end{aligned}
$$

\section{B. Integration of Equation 39}

The integral is split into its additive components. The observation was made by Stewart [4] on page 122 that

$$
\frac{d}{d x} \tan x=\sec ^{2} x
$$

On the inside back cover of Stewart [4] it is observed that Eq. 71 of his Table of Integrals has

$$
\int \sec ^{3} u d u=\frac{1}{2} \sec u \tan u+\frac{1}{2} \log |\sec u+\tan u|+C
$$

Finally it is known that

$$
\int \sec x d x=\log |\sec x+\tan x|
$$

The logarithmic term in Eq. 57, the integral of the cube of the secant, is subtracted from the last-mentioned integral, and this results in Eq. 40.

\section{REFERENCES}

[1] Bryson, A. E. AND Y.-C. Ho, Applied Optimal Control: Optimization, Estimation and Control, John Wiley \& Sons (Hemisphere Publishing Co.), New York, revised (2nd) ed., 1975.

[2] Pontryagin, L. S., V. G. Boltyanskit, R. V. Gamkrelidze and E. F. MishsCHENKO, The mathematical theory of optimal processes, Interscience Publishers, London, 1962. Authorized translation from the Russian, translator K. N. Trirogoff.

[3] Smith, R., Y. ChaO, B. H. Jones and G. S. Sukhatme, Towards the improvement of autonomous glider navigational accuracy through the use of regional ocean models. ASME Ocean, Offshore and Arctic Engineering Division OMAE2010-21015, 2010. The 29th International Conference on Ocean, Offshore and Arctic Engineering Shanghai, China - June 6-11, 2010.

[4] Stewart, J., Calculus, Brooks/Cole Publishing Company, Pacific Grove, CA, 1987.

[5] ZERMELO, E., Über das Navigationsproblem bei Ruhender oder veränderlicher Windverteilung, Zeitschrift für Angewandte Mathematik und Mechanik (ZAMM), 11 (1931), pp. 114-124.

[6] Zlobec, S., Partly convex programming and Zermelo's navigation problems, Journal of Global Optimization, 7 (1995), pp. 229-259. 


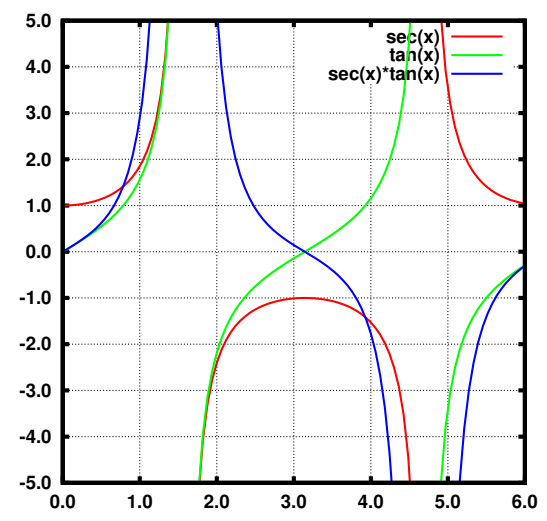

(a) Some useful trigonometric graphs.

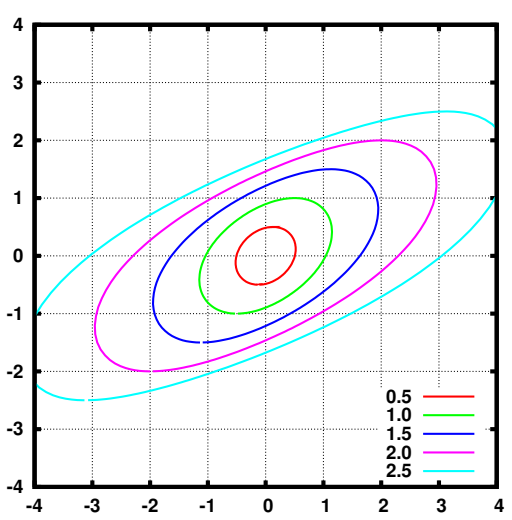

(c) Constant time contours

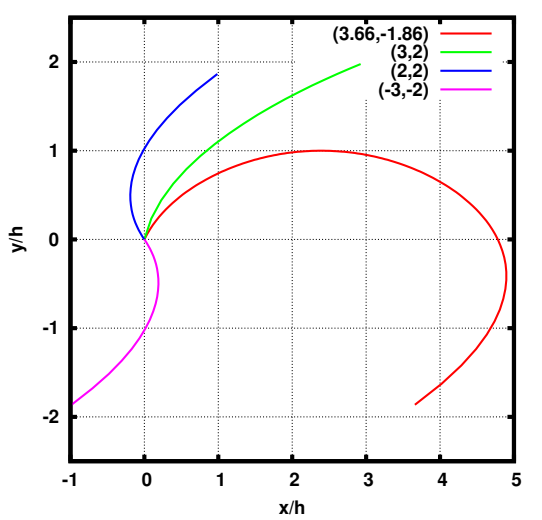

(b) Various paths of minimum time navigation.

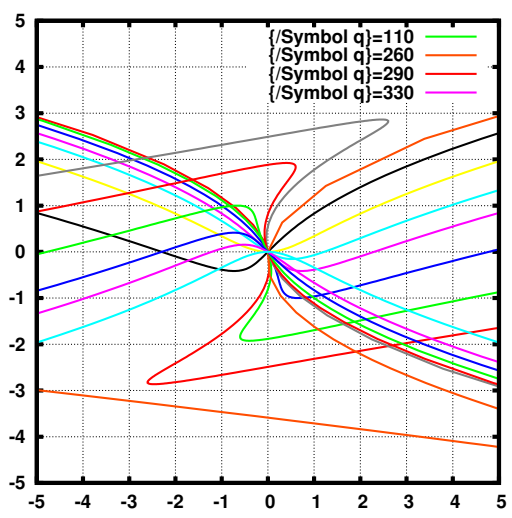

(d) Various paths of constant-course angle.

Fig. 1. Figures for 'Autonomous Underwater Vehicle Minimum-Time Navigation in a Current Field'. 